\title{
4 Digitale Versorgung für chronisch Kranke
}

\author{
Jasper zu Putlitz
}

\section{Chronisch Kranke müssen über Sektorengrenzen hinweg besser versorgt werden}

Eine typische Episode: Die allein lebende 74-jährige Rentnerin Erika S. leidet seit Jahren an Bluthochdruck, Diabetes und Herzinsuffizienz. Wegen akuter Atemnot wurde sie vor 10 Tagen über die Notaufnahme ihres lokalen Krankenhauses stationär aufgenommen und wird heute in deutlich verbessertem Zustand stabilisiert nach Hause entlassen. Ihre chronische, offene Wunde am Bein wurde während des stationären Aufenthaltes versorgt, erfordert aber weitere intensive pflegerische Betreuung. Der Sozialdienst des Krankenhauses informiert den ambulanten Pflegedienst. Die Entlassdokumentation wird der Patientin mitgegeben, ist aber für sie schwer verständlich. Trotz deutlicher Bewegungseinschränkung muss sich die Rentnerin aus der Apotheke Verbandmaterial, aus dem Sanitätshaus Stützstrümpfe besorgen. Die in der Nähe lebende Tochter der Patientin, selbst berufstätig, versucht, ihre Mutter zu unterstützen, aber ihr fehlen Informationen und sie weiß nicht, wen sie fragen kann - für Patientin und Angehörige eine sehr stressige Situation.

Viele Patienten, die aus dem Krankenhaus entlassen werden, müssen sich im Dschungel der Zuständigkeiten im Gesundheitssystem zurechtfinden. Aus Sicht der Patienten und ihrer Angehörigen ist das deutsche Gesundheitswesen mit seiner Vielzahl von Akteuren und Vorschriften oft sehr unübersichtlich. Die einzelnen Sektoren arbeiten häufig isoliert voneinander und ohne patientenfokussierte Koordination. Patienten und ihre Angehörigen fühlen sich leicht verloren - und das ausgerechnet in Phasen wie oben beschrieben, in denen sie sich vor allem um die Gesundheit und 
nicht um das Finden des nächsten Ansprechpartners kümmern sollten. Eine Accenture-Studie mit mehr als 1.000 Patienten in Deutschland belegt, dass sich $79 \%$ der Befragten einen einzigen Ansprechpartner wünschen, der ihnen Informationen und Ratschläge für die Gesundheit gibt $-72 \%$ der Befragten bevorzugen dabei ihren Arzt, der aber oft zu wenig Zeit hat, ausführliche Informationsgespräche zu führen. Nahezu zwei Drittel der Befragten gaben zudem an, mehr und zeitgerechtere Informationen zur ihrer Erkrankung haben zu wollen - insbesondere in der Zeitperiode zwischen Auftreten von Symptomen und Diagnose.

Gerade für Patienten mit chronischen Erkrankungen wie Diabetes, Herzinsuffizienz und chronisch-obstruktiver Lungenerkrankung (COPD) kommt es beim Sektorübergang von der stationären Behandlung in die ambulante Versorgung auf Kontinuität und Koordination der Versorgung an. Hier müssen Versorgungssektoren überwunden werden - und das auf kontinuierlicher Basis, denn mit fortschreitendem Krankheitsverlauf steigt die Wahrscheinlichkeit von Komplikationen und damit verbundenen, oft ungeplanten Krankenhausaufenthalten (oft Wiederaufnahmen innerhalb kurzer Zeit nach Entlassung). Ein Großteil der im Gesundheitssystem anfallenden Kosten ist zudem mit chronischen Krankheiten assoziiert. Auch deswegen versucht der Gesetzgeber im Rahmen der letzten Gesundheitsreformen seit Jahren, eine stärkere Vernetzung der verschiedenen Sektoren und Koordination der Versorgung zu erreichen. Mit dem GKV-Versorgungsstärkungsgesetz wurden neue Rahmenbedingungen für eine sektorenübergreifende, flächendeckende und damit gut zugängliche medizinische Versorgung geschaffen und das Entlassmanagement aus dem Krankenhaus gestärkt. Die kürzlich in Kraft getretenen Pflegestärkungsgesetze führen zu weitreichenden Verbesserungen in der Versorgung Pflegebedürftiger, insbesondere auch im ambulanten Bereich.

\section{Digitale Transformation in der lokal koordinierten Versorgung}

Nicht nur der Gesetzgeber ist bestrebt, Schnittstellen zu optimieren und Leistungserbringer über die Sektoren hinweg enger zusammen zu bringen. Auch einzelne Akteure versuchen, Sektorengrenzen zu überwinden. Eine geeignete Möglichkeit sind regional oder lokal koordinierte Netzwerke, die den Patienten und dessen optimale Versorgung in den Mittelpunkt des Handelns stellen. Hierzu ist es notwendig, den Weg des einzelnen Patienten als einen kontinuierlichen Prozess zu sehen, von der Krankenhauseinweisung über das Entlassmanagement bis hin zur häuslichen oder Heimversorgung. Ziel eines auf die Versorgung von chronisch Kranken ausgerichteten Netzwerkes ist es, die klassischen Sektorengrenzen zu überwinden und den gesamten Patientenweg abzubilden. Dadurch kann für den Patienten eine Verbesserung der Lebensqualität ermöglicht werden, und seine Angehörigen profitieren ebenfalls davon.

An einer koordinierten Versorgung chronisch Kranker sind viele Akteure beteiligt, die verschiedene Produkte und Dienstleistungen beitragen und über die unterschiedlichsten Prozesse und Systeme verfügen, die oft primär auf rechtliche Konformität und die Belange der Dokumentation und Abrechnung, nicht jedoch auf die Patientenbetreuung per se ausgerichtet sind. Dies macht Versorgungskoordination derzeit noch zu einer großen Herausforderung. 
Wie die digital unterstützte, koordinierte Versorgung chronisch Kranker funktioniert, zeigt das Beispiel eines internationalen Anbieters von Medikamenten und Ernährungsprodukten. Gemeinsam mit Netzwerkpartnern, die wichtige, ergänzende Produkte, Dienstleistungen und Kernkompetenzen einbringen, wird das Unternehmen in seinen Märkten regional und lokal zukünftig koordinierende Leistungen in der Versorgung chronisch Kranker übernehmen und auf diese Weise einen Beitrag zur verbesserten Versorgung leisten.

Dafür entwickelten Accenture und Fjord gemeinsam mit dem Unternehmen eine innovative Digitalplattform, die eine effiziente Koordination des Versorgungsprozesses rund um den Patienten, von der Aufnahme und der Betreuung zu Hause bis hin zur Logistik und Abrechnung der Leistungen ermöglicht. Kürzere Entscheidungswege, klare Verantwortlichkeiten, reduzierte Schnittstellen und ein ganzheitlicher Blick auf den gesamten Behandlungsweg kommen dem Patienten direkt zugute. Die Vision: der chronisch kranke Patient verfügt über einen digitalen Gesundheitslotsen.

Für die Realisierung dieser ambitionierten digitalen Transformation wurden neue Wege in der Entwicklung beschritten. Gemeinsam traten die Entwicklungspartner zu Design-Thinking-Workshops an, um mit Kreativität, Technologie und Fachwissen den Weg des Patienten durch das Gesundheitssystem aufzuzeichnen. Krankenschwestern und Patientenkoordinatoren aus mehreren Ländern trugen als Nutzer der Lösung ihre vielfältigen individuellen Erfahrungen über Anforderungen und Bedürfnisse der Patienten und ihrer Angehörigen bei - denn die Perspektive der Patienten und der Nutzer sind beide notwendig, um die Herausforderungen einer guten Versorgung von chronisch Kranken zu lösen.

Die innovative, als Medizinprodukt zertifizierte, digitale Versorgungsplattform entstand in enger Abstimmung mit allen Beteiligten entlang der gesamten Patientenreise. Individuelle Patientenpfade dienten als Ausgangspunkt für die Entwicklung von sog. „User Stories“, die auf ein Prozessmodell bezogen und von agil arbeitenden Softwareentwicklungs-(„Scrum“-)Teams in die modulare Programmierung der Lösung übersetzt wurden. Die Digitalplattform verfügt zudem über eine moderne und sichere Cloud-Architektur, die die Versorgungskoordination zu jeder Zeit und überall unterstützt und einen sehr hohen Grad von Datenschutz gewährleistet.

\section{Mehrwert digitaler Versorgungskoordination}

Die Tablet-basierte Lösung bedeutet für Patienten und Nutzer einen großen Fortschritt. Alle Informationen über den Patienten sind schnell und übersichtlich verfügbar. Mit wenigen Schritten auf dem Touchscreen kann die Zuteilung eines Betreuenden zu einem Patienten erfolgen. Mit Einwilligung des Patienten besteht die Möglichkeit, einen Befund, z.B. eine Wunde, für die weitere Beurteilung fotografisch zu dokumentieren. Produktbestellungen können sofort ausgelöst und weiterbearbeitet werden. Für den Patienten bedeutet dies: Schnelle und zielgerechte Versorgung, auch mit Produkten von Partnern - und eine hohe Dokumentationsqualität, die der weiteren, gut koordinierten Versorgung zugutekommt. Betreuer profitieren von deutlich gesteigerter Effizienz in ihrem Arbeitsalltag und haben so mehr Zeit am Patienten. Erfolgskritisch ist die sehr gute „User Experience“ die Lösung, die die Akzeptanz bei den Nutzern sichert. Durch frühzeitige Einbindung der Nutzer wurde gewähr- 
leistet, dass alle Funktionalitäten den Alltag der Nutzer vollständig abbilden. Die intuitive Bedienoberfläche minimiert darüber hinaus den Schulungs- und Trainingsaufwand.

\section{Digitale Versorgung - Trends und Entwicklung}

Die Fähigkeit, die Chancen der Digitalisierung mit dem Mehrwert für Patienten und alle an seinem Versorgungsprozess Beteiligten zu kombinieren, ist eine Kernkompetenz für Unternehmen im Gesundheitswesen der Zukunft. Die neue Digitalplattform ist ein erster wichtiger Beitrag des Unternehmens auf dem Weg zu einer besseren, intersektoral koordinierten Patientenversorgung aus einer Hand und ermöglicht über Versorgungssektoren hinweg eine bessere Zusammenarbeit mit anderen Dienstleistern und Herstellern. In Zukunft werden Datenströme mit patientenrelevanten Informationen noch stärker integriert sein - beispielsweise durch Schnittstellen zu Krankenhaus-Informationssystemen und Arzt-Informationssystemen sowie zum Patienten selbst. Mit neuen Entwicklungen in der Sensorik können chronisch Kranke auch zu Hause durch Monitoring ihrer Vitalparameter besser betreut werden, damit eine sich abzeichnende Verschlechterung frühzeitig erkannt wird. Die teil-automatisierte Analyse von großen Datenströmen wird hier eine entscheidende Rolle spielen. Daraus ergibt sich auch die Möglichkeit, die Ergebnisse der Behandlung zu einem maßgeblichen Qualitäts- und Vergütungsindikator zu entwickeln.

\section{Praxistipps}

- Digitalisierung als Chance für Prozessoptimierung nutzen: Der Übergang auf eine digitale Lösung bietet eine einmalige Chance, Prozesse auf ihre Sinnhaftigkeit und Effizienz zu überprüfen und gegebenenfalls anzupassen. Nicht allen Prozess-Ballast digitalisieren!

- Nutzer von Anfang an in den Entwicklungsprozess einbinden: Am Anfang Zeit investieren, um die Welt aus den Augen des zukünftigen Nutzers und seiner Interaktionspartner zu verstehen. Nutzer aktiv am Design-Prozess beteiligen!

- Agil arbeiten - „fail fast“: Häufige Nutzerrückkopplung in den Entwicklungprozess einbauen und Korrekturen rasch umsetzen. Ausreichend Zeit für Schulung und Kommunikation einplanen! 